\title{
AJSE Relaunched in Cooperation with Springer
}

Published online: 11 January 2011

(C) King Fahd University of Petroleum and Minerals 2011

The Arabian Journal for Science and Engineering (AJSE) is a well-established, peer-reviewed, and ISI journal published and owned by the King Fahd University of Petroleum \& Minerals (KFUPM) (Dhahran, Saudi Arabia) since 1975. We are happy to announce that starting January 2011, AJSE will be published by the prestigious international publisher, Springer.

Springer will also market and distribute the journal as part of its program to further increase the global circulation and the impact of the journal.

The Springerlink.com platform delivers the contents of the AJSE to a truly regional and global audience. The new and enhanced online submission provides access to additional information and discoverability that is aided by related article and semantic linking.

The journal's aims and scope provide a broad range of coverage that includes the Science disciplines of Chemistry, Earth Sciences, Physics, and the Mathematical Sciences disciplines, in addition to the Engineering disciplines of Civil, Chemical, Electrical, Mechanical, Petroleum Engineering, Computer and Systems Engineering, together with Themed Special Issues on specific topics.

As the AJSE begins its 36th year, we are enjoying an increase in readership and in quality manuscript submissions. Due to the large amount of submissions, the AJSE publishes now eight issues per year of rigorous and original contributions. Furthermore, we are including more articles in each issue in order to minimize the delay of publication of the large number of accepted papers.

A more than $100 \%$ increase in the articles submitted in 2010, compared to the submissions in the year 2008 , demonstrates that an increasing number of regional and international authors are recognizing the quality of AJSE, supported by peer reviewing of a high standard that focuses and builds upon the constructive aspects of the research results included in the submitted manuscript.

We are also very proud to present our new journal design that consists of three sections: AJSE-Engineering, AJSE-Science, and AJSE-Mathematics.

We strongly encourage researchers and scientists worldwide to submit their high-quality articles for consideration of publication in AJSE. We have established an energetic and dedicated editorial board and office to ensure that your article will be reviewed and published rapidly.

B. M. El Ali (殴

King Fahd University of Petroleum and Minerals, P. O. Box 5033,

Dhahran 31261, Saudi Arabia

E-mail: belali@kfupm.edu.sa 